\title{
A COMPARISON ON SPECKLE NOISE SUPPRESSION ALGORITHM FOR INTRAVASCULAR ULTRASOUND (IVUS) IMAGES
}

\author{
K V Archana \\ Assistant Professor, Department of Electronics and Communication Engineering, Avinashilingam Institute for \\ Home Science and Higher Education for Women, \\ Coimbatore, Tamil Nadu, India \\ archana_ece@avinuty.ac.in
}

\section{R. Vanithamani}

Professor, Department of Biomedical Instrumentation Engineering, Avinashilingam Institute for Home Science and Higher Education for Women, Coimbatore, Tamil Nadu, India vanithamanibmie@avinuty.ac.in

\begin{abstract}
Accurate and reliable diagnosis of carotid artery stenosis depends on the quality of IVUS images. Especially in ultrasound images where coherent sources are involved, speckle noise causes blurring and loss of information. Thus, methods to eliminate speckle noise plays an essential part in the field of medical imaging. This paper compares various speckle noise suppression algorithms for carotid artery ultrasound images. Speckle noise reduction algorithms that are implemented includes Homomorphic Wavelet Level 1 and Level 2, Perona-Malik (PM) filter, Modified PM1, Modified PM2, Adaptive PM, Butterworth Filter, Doubly Degenerative Diffusion (DDD), Speckle Reducing Anisotropic Diffusion (SRAD) and Total Variance (TV) filter. A quantitative evaluation is carried out by estimating Signal to Noise Ratio (SNR), Peak Signal to Noise Ratio (PSNR), Structural Similarity Index (SSIM), Beta Metric and Natural Image Quality Evaluator (NIQE). The performance metrics shows that Homomorphic Wavelet Level1, Modified PM 2, Adaptive PM and SRAD are robust in eliminating speckle noise from carotid artery ultrasound images, thereby increasing its diagnostic accuracy. Though DDD and TV approach have good SNR and PSNR values, their low Beta metric and high NIQE values have made them ineffective.
\end{abstract}

Keywords: Speckle noise reduction; intravascular ultrasound images; speckle reducing anisotropic diffusion; Perona-Malik filter.

\section{Introduction}

Carotid arteries are major blood vessels that supply blood to head and brain. Narrowing of such arteries due to plaque formation is known as carotid artery stenosis. In some instances, it can advance to complete blockage leading to stroke. So, imaging of carotid artery plays a vital role in diagnosis of carotid artery stenosis and to quantitatively monitor carotid plaque progression during the course of treatment. Over some years, ultrasound imaging has been successfully utilized for arterial disease diagnosis due to its non-invasive nature and low cost. Speckle pattern often has negative effect on the quality of ultrasound images, which may cause poor diagnosis. Though speckle is considered as a noise, it carries some useful information. But still, it limits the contrast resolution of imaging modality and the efficiency of diagnosis. Therefore, speckle pattern should be suppressed without affecting important features of the image. The aim of this work is to give an extensive comparison on various algorithms used for despeckling carotid artery ultrasound images. This paper is structured as follows: Section 2 discusses some of the related works, Section 3 elaborates various despeckling algorithms to be compared, Section 4 gives the experimental results and Section 5 concludes the paper. 


\section{Related work}

Speckle noise suppression technique is an essential pre-processing step because the presence of speckle pattern may disturb the feature extraction and analysis processes. Over past few years, numerous researchers have developed various algorithms ranging from simple filters to complicated algorithms to suppress speckle noise. Some commonly known spatial domain despeckling filters such as Lee, Kuan, Frost, median and Weiner are utilized in [1], [2] and [3]. In [4] and [5] Bilateral and Gaussian filters are implemented for speckle reduction in ultrasound. In recent years filtering in wavelet domain has become popular among researchers. Various kinds of wavelets such as Haar, Daubechies, Symlets, Coiflets and Biorthogonal are utilized for despeckling ultrasound images in [6], [7], and [8]. Some used thresholding in wavelet domain as in [9] [10] which proved to be effective. Recently, combination of algorithms is prevalently adopted in denoising ultrasound images. For example, an optimization algorithm cascaded with Weiner filter in [11], combination of wavelet and enhanced Kuan filter in [12], and combination of wavelet and bilateral filter in [13]. Most of the despeckling algorithms have certain demerits that has to be addressed. Some despeckling methods which use windows can have variation in its output for different window sizes [14]. Some methods require threshold values to be specified in the filtering process that should be estimated empirically. Wherein, inappropriate choice of threshold may lead to noisy boundaries. There are several papers which discusses about the despeckling algorithms for Synthetic Aperture Radar (SAR) images, satellite images, Optical Coherence Tomography (OCT) images and fetal ultrasound. But a comparison using carotid artery ultrasound is very limited. Table.1.1. summarizes works done on comparison of despeckling algorithms specifically for carotid artery ultrasound images in recent years.

\begin{tabular}{|l|c|c|}
\hline \multicolumn{1}{|c|}{ Author/Paper } & No. of algorithms compared & Comparison metrics \\
\hline Rafati, M., et. al. [15] & 3 & 10 \\
\hline Nieniewski, M., et. al. [16] & 16 & 1 \\
\hline Loizou, C.P., et al. [17] & 10 & 9 \\
\hline Yu, Y., [22] & 3 & 3 \\
\hline
\end{tabular}

Table 1. Summary of works comparing despeckling algorithms for carotid artery ultrasound

Although numerous despeckling algorithms have been proposed in the literature, a systematic comparison on the performance of these filters is still very beneficial to facilitate the selection of appropriate filter for clinical application. This work focuses on comparing the performance of ten filters for suppressing speckle noise in carotid artery ultrasound images.

\section{Despeckling filters}

\subsection{Butterworth filter}

Butterworth filters are more common in signal processing domain. In image processing applications, it is used for smoothing in frequency domain. As the speckle noise is present in high frequency, this filter removes high frequencies and preserves the low frequency components [14].

\subsection{DDD filter}

DDD algorithm was developed by Zhengyi Zhou et al in [19] and found to be inspired by non-linear diffusion models. This algorithm was noted to denoise rapidly due to implementation of slightly modified Fast Explicit Diffusion scheme. This framework is capable of boosting the overall efficiency by several times.

\subsection{Homomorphic wavelet filter}

In homomorphic wavelet filtering, input image undergoes logarithmic transform, followed by discrete wavelet transform. With the help of wavelet coefficients, inverse discrete wavelet transform is performed to get a reconstructed image. The exponential of this reconstructed image is the filtered output [18]. In this work, filtering is done in two levels. In level 1 filtering, input image is split into four sub bands as LL, LH, HL and $\mathrm{HH}$. Since, most of the speckle noise is present in HH band, it is eliminated. In level 2 filtering, the entire process is repeated with level 1 filtered image as input.

\subsection{SRAD filter}

Speckle reducing anisotropic diffusion filter often known as SRAD is an extended version of anisotropic diffusion filter designed by $\mathrm{Yu}$ and Acton in [22]. SRAD distinguishes the edges in the images using instantaneous coefficient of variation, which provides high values for edges and low values in homogenous regions. The high values decrease the smoothing effect in edges; hence edges are preserved to a greater extent. Whereas speckle pattern is removed in homogenous regions [20]. 


\subsection{PM filter}

It is a non-linear anisotropic diffusion method proposed by Perona and Malik in 1990 that uses techniques of partial differential equations. Later on, many variations and improvements have been made to this filtering process. In this work, four different versions of Perona Malik filter such as PM, Modified PM 1, Modified PM 2 and Adaptive PM proposed by Anthony Bua [21] are utilized whose equations are as follows.

\subsubsection{PM Filter}

$$
\begin{aligned}
& v_{X}=1 \hat{f}\left(1+\left(c a b s\left(c_{x}\right) / E_{k}, \wedge 2\right)\right. \\
& \left.\mathbb{P M}=1+d_{E} *\left(\varepsilon_{W} * C_{W}+z_{S} * C_{S}+\varepsilon_{E} * C_{E}+z_{W} * C_{W}\right)-\text { lambda } * Q\right)
\end{aligned}
$$

\subsubsection{Modified PM1}

$$
\boldsymbol{z}_{X}=1 /\left(1+\left((a b s)\left(C_{x}\right)\right) / E_{1}\right)+\left(\left(a b s\left(C_{X}\right)\right) / E_{2}\right) / n
$$

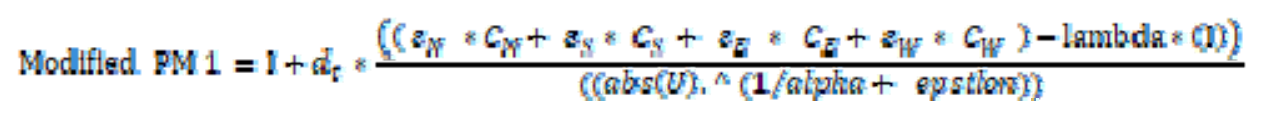

\subsubsection{Modified PM2}

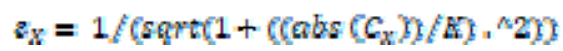

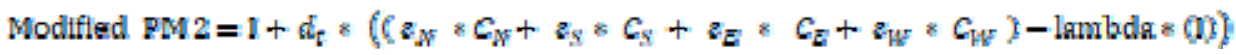

3.5.4. Adaptive $P M$

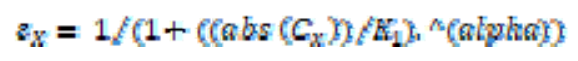

$$
\text { Adlaptixe } \left.P M=1+d_{E} *\left(\varepsilon_{W} * C_{W}+z_{S} * C_{S}+z_{E} * C_{E}+z_{W} * C_{W}\right)-\text { lambda } * Q\right)
$$

Where,

$\mathrm{x}$ denotes directions (North, East, West and South),

$\mathrm{Cx}$ denoted change in different directions;

$\mathrm{K}, \mathrm{K} 1$ and $\mathrm{K} 2$ are the shape defining constant; alpha and epsilon are constants; $\mathrm{dt}$ is the time interval between space solutions and lambda is the regularization constant; $\mathrm{I}$ is the noisy image.

\subsection{TV model}

TV denoising approach is developed with an aim to preserve sharp edges while smoothing homogenous regions. The regularization parameter controls the degree of smoothing effect. Numerous TV based denoising algorithms have been developed. This work implements an algorithm proposed by Anthony Bua in [21]. The mathematical equation of Total Variance Regularization is given as:

$$
T(w)=F\left(b / A_{u}\right)+\lambda\|\Delta w\|
$$

Where, $\mathrm{F}$ is the data fidelity term, which depends on the noise model and $\lambda$ is the regularization parameter. The multiplicative model is represented as:

$$
b=\left(A_{u}\right){ }_{k} g_{2}
$$

Where, $\mathrm{A}$ is the observation operator, $\mathrm{u}$ is the noise free data and $\zeta$ is the noise 


\section{Results}

Ten despeckling algorithms are implemented on IVUS images and their output is shown in Fig.1.2.

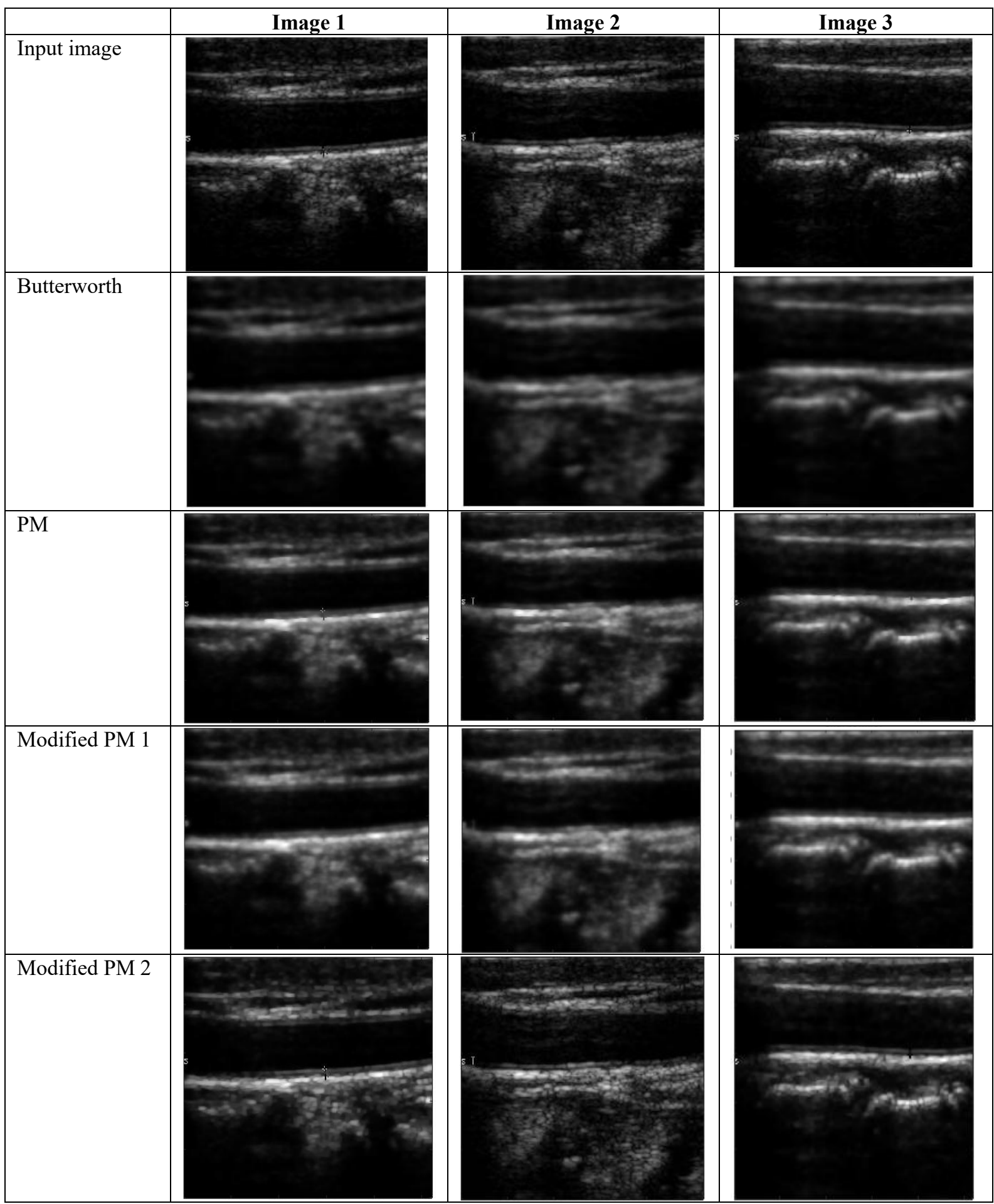




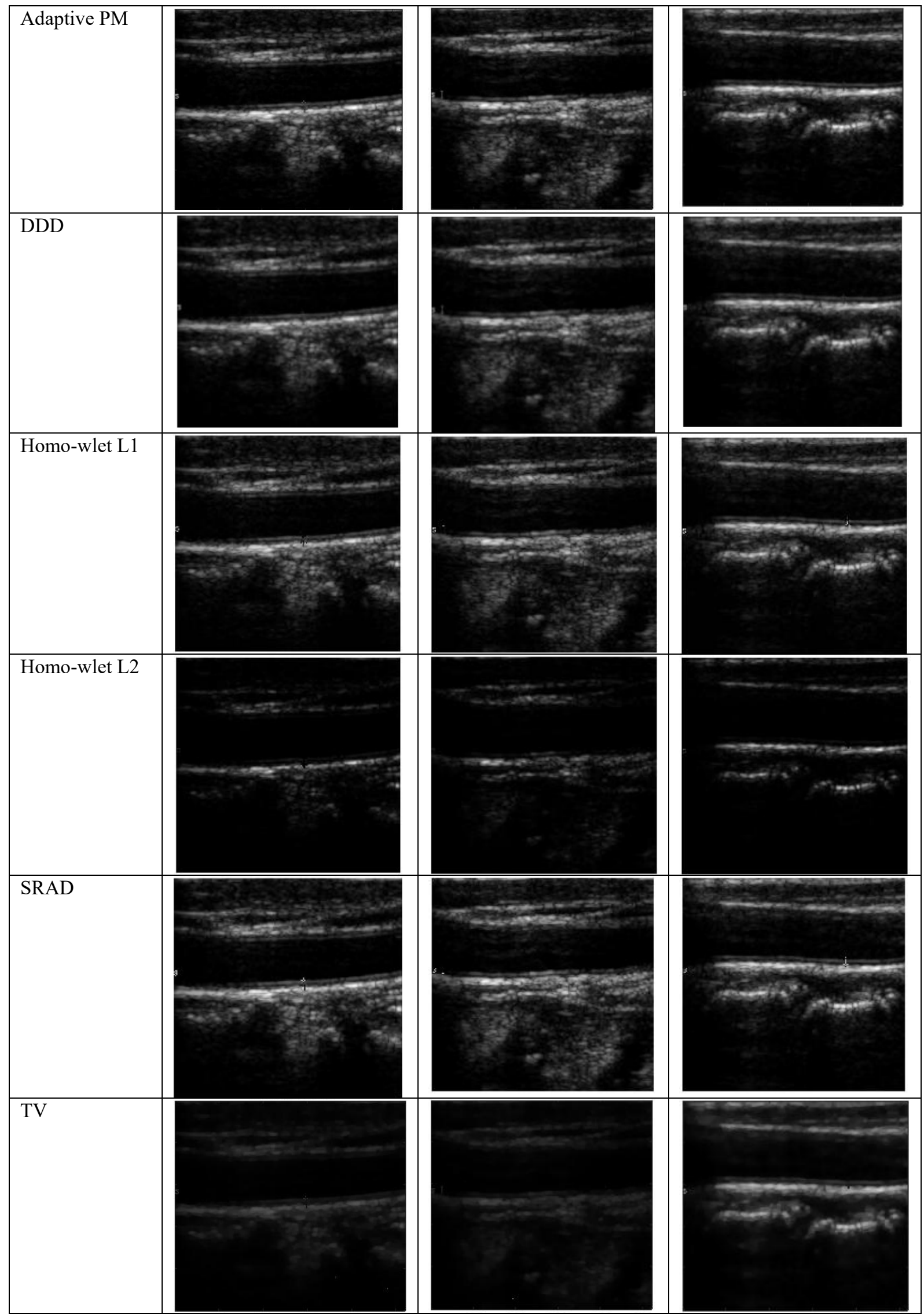

Figure.1.2. Resultant images of various despeckling algorithms 
Despeckling algorithms are compared in terms of five image quality metrics such as Signal to Noise Ratio (SNR), Peak Signal to Noise Ratio (PSNR), Structural Similarity Index Measure (SSIM), Beta Metric and Natural Image Quality Evaluator (NIQE). PSNR expresses the ratio of maximum possible value of image and power of noise in range of decibels. SSIM provides comparison between two images based on luminance, contrast and structure. Therefore, this metric takes into account the factors of human visual system. NIQE is a completely blind image quality score which does not require any reference image. A lower NIQE score signifies or denotes better perceptual quality. Beta Metric value indicates the edge preserving condition in the image and its value ranges between -1 to +1 . Table 2 . Shows the values of the metrics that are calculated for all the ten filters.

\begin{tabular}{|c|c|c|c|c|c|}
\hline \multicolumn{6}{|c|}{ Image 1} \\
\hline & SNR (dB) & PSNR (dB) & SSIM & Beta metric & NIQE \\
\hline Butterworth & 17.0268 & 28.2049 & 0.7722 & 0.0581 & 5.4423 \\
\hline Homo-wavelet L1 & 22.4286 & 32.8915 & 0.9684 & 0.7062 & 4.5548 \\
\hline Homo-wavelet L2 & 12.2472 & 19.6408 & 0.4440 & 0.2125 & 6.4660 \\
\hline PM & 17.3775 & 29.7711 & 0.8165 & 0.7269 & 5.2345 \\
\hline Modified PM1 & 16.9664 & 28.9839 & 0.8087 & 0.4784 & 5.4375 \\
\hline Modified PM2 & 19.2578 & 36.2369 & 0.9134 & 0.8865 & 4.6470 \\
\hline Adaptive PM & 21.2722 & 39.6659 & 0.9848 & 0.6512 & 4.2170 \\
\hline DDD & 22.6201 & 32.2041 & 0.9492 & 0.2414 & 5.7471 \\
\hline SRAD & 20.9932 & 31.7674 & 0.8960 & 0.9678 & 4.2345 \\
\hline TV & 20.0979 & 33.4916 & 0.8682 & 0.4795 & 5.5907 \\
\hline \multicolumn{6}{|c|}{ Image 2} \\
\hline & SNR & PSNR & SSIM & Beta metric & NIQE \\
\hline Butterworth & 16.8482 & 27.9927 & 0.7459 & 0.0703 & 5.7619 \\
\hline Homo-wavelet L1 & 22.9840 & 33.1328 & 0.9790 & 0.7363 & 4.5287 \\
\hline Homo-wavelet L2 & 11.8141 & 19.1700 & 0.3735 & 0.3053 & 5.7325 \\
\hline PM & 17.0912 & 29.4471 & 0.7940 & 0.7640 & 5.3577 \\
\hline Modified PM1 & 16.0410 & 28.7110 & 0.7866 & 0.4987 & 5.2755 \\
\hline Modified PM2 & 19.9502 & 35.5860 & 0.9017 & 0.8454 & 4.7071 \\
\hline Adaptive PM & 21.6993 & 40.0552 & 0.9832 & 0.6851 & 4.8266 \\
\hline DDD & 23.8979 & 32.1440 & 0.9397 & 0.2489 & 5.6164 \\
\hline SRAD & 21.0301 & 32.7303 & 0.7939 & 0.9538 & 4.9373 \\
\hline TV & 19.9804 & 32.3363 & 0.8466 & 0.3228 & 4.7793 \\
\hline \multicolumn{6}{|c|}{ Image 3} \\
\hline & SNR & PSNR & SSIM & Beta metric & NIQE \\
\hline Butterworth & 17.9540 & 28.4858 & 0.8048 & 0.0583 & 5.4984 \\
\hline Homo-wavelet L1 & 21.9564 & 32.1224 & 0.9492 & 0.7085 & 4.1570 \\
\hline Homo-wavelet L2 & 12.4526 & 19.5022 & 0.3456 & 0.3025 & 5.4028 \\
\hline PM & 17.1324 & 28.2473 & 0.7041 & 0.7242 & 6.4336 \\
\hline Modified PM1 & 16.0410 & 29.8052 & 0.8561 & 0.4673 & 5.2894 \\
\hline Modified PM2 & 19.6004 & 35.7642 & 0.9056 & 0.8341 & 5.0543 \\
\hline Adaptive PM & 20.2577 & 39.1341 & 0.9723 & 0.6429 & 4.4003 \\
\hline DD & 23.4903 & 32.5726 & 0.9557 & 0.2184 & 5.3332 \\
\hline SRAD & 20.8342 & 31.2741 & 0.8242 & 0.9619 & 5.4302 \\
\hline TV & 20.9804 & 32.4317 & 0.8904 & 0.3109 & 5.2967 \\
\hline
\end{tabular}

Table 2. Performance metrics

\section{Conclusion}

This work presents a comparative analysis on different despeckling methods with an aim to suppress the speckle noise in carotid artery ultrasound in order to make the images suitable for further analysis. The experimental results show that Homomorphic Wavelet Level1, Modified PM 2, Adaptive PM, SRAD and DDD filtered images obtained higher SNR and PSNR values indicating successful despeckling. SSIM values are higher for Adaptive PM and Homomorphic Wavelet Level 1. Beta metric is higher for SRAD and Modified PM 2 which shows that edges are preserved. Whereas, Butterworth, Homomorphic Wavelet Level2 and DDD did not preserve the edges resulting in blurred image. Further, NIQE values indicate that Adaptive PM and SRAD maintained the perceptual quality of image successfully. Overall, this work gives a clear picture on performance of various despeckling algorithms which can be utilized in development of diagnostic systems. Future work will focus on critical analysis of deep learning methods for despeckling applications. 


\section{References}

[1] Saranya, M.; and Saraswathy, C. Speckle Reduction in Ultrasound Image. International Journal of Electronics and Computer Science Engineering, ISSN: 2277-1956, 1(2), pp. 343-347.

[2] Sivakumar, R.; Gayathri, M. K; and Nedumaran, D. (2010): Speckle filtering of ultrasound B-Scan Images - a comparative study between spatial and diffusion filters, 2010 IEEE Conference on Open Systems (ICOS 2010). Kuala Lumpur, Malaysia, pp. 80-85.

[3] Mohan, R.; Mridula, S.; and Mohanan, P. (2016): Speckle noise reduction in images using Wiener filtering and adaptive Wavelet thresholding. 2016 IEEE Region 10 Conference (TENCON). Singapore, pp. 2860-2863.

[4] Balocco, S.; Gatta, C.; Pujol, O.; Mauri, J.; and Radeva, P. (2010): SRBF: Speckle Reducing Bilateral Filtering. Ultrasound in medicine \& biology. 36: 1353-63. 10.1016/j.ultrasmedbio.2010.05.007.

[5] Parminder, P. K.; and Tejinderpal, S. (2014): Speckle Noise Reduction in Ultrasound Images: Performance Analysis and Comparison. International Journal of Engineering Research \& Technology, 3(7).

[6] Lazrag, H.; and Naceur, M. S. (2012): Wavelet filters analysis for speckle reduction in intravascular ultrasound images. 2012 6th International Conference on Sciences of Electronics, Technologies of Information and Telecommunications (SETIT).

[7] Yadav, A. K.; Roy, R.; Kumar, A. P.; Kumar, C. S.; and Dhakad S. K. (2015): De-noising of ultrasound image using discrete wavelet transform by symlet wavelet and filters. 2015 International Conference on Advances in Computing, Communications and Informatics (ICACCI), Kochi, India, pp. 1204-1208.

[8] Chinnathambi, V.; Sankaralingam, E.; Thangaraj, V.; and Padma, S. (2019): Despeckling of ultrasound images using directionally decimated wavelet packets with adaptive clustering. IET Image Processing, 13(1), pp. 206-215.

[9] Vanithamani, R.; and Umamaheswari, G. (2011): Wavelet based despeckling of medical ultrasound images with bilateral filter. TENCON 2011 - 2011 IEEE Region 10 Conference. Bali, Indonesia, pp. 389-393.

[10] Randhawa, S.K.; Sunkaria, R.K; and Puthooran, E. (2019). Despeckling of ultrasound images using novel adaptive wavelet thresholding function. Multidim Syst Sign Process, 30, pp. 1545-1561.

[11] Dass, R. (2018): Speckle Noise Reduction of Ultrasound Images Using BFO Cascaded with Wiener Filter and Discrete Wavelet Transform in Homomorphic Region. Procedia Computer Science, 132, pp. 1543-1551.

[12] Akl, A.; and Yaacoub, C. (2013): A hybrid filter for image despeckling with wavelet-based denoising and spatial filtering. 2013 Third International Conference on Communications and Information Technology (ICCIT). Beirut, Lebanon, pp. 325-329.

[13] Olfa, M.; and Nawres, K. (2014): Ultrasound image denoising using a combination of bilateral filtering and stationary wavelet transform. International Image Processing, Applications and Systems Conference. Sfax, Tunisia, pp. 1-5.

[14] Loizou, C. P.; Pattichis, C. S.; Christodoulou, C. I.; Istepanian, R. S. H.; Pantziaris, M.; and Nicolaides, A. (2005): Comparative evaluation of despeckle filtering in ultrasound imaging of the carotid artery. IEEE Transactions on Ultrasonics, Ferroelectrics and Frequency Control, 52(10), pp. 1653-1669.

[15] Rafati, M.; Arabfard, M.; Rafati M.R.; Voshtani, H.; and Moladoust, H. (2015): A Comparative Study of Three Speckle Reducing Methods for Intima-Media Thickness Ultrasound Images, Iran, Red Crescent Med. J, 17(2), pp. 1-8.

[16] Nieniewski, M.; and Zajączkowski, P. (2017): Comparison of Ultrasound Image Filtering Methods By Means of Multivariable Kurtosis. Image Anal. Stereol, 36(2), pp. 79-85.

[17] Loizou, C.P.; Pattichis, C. S.; Christodoulou, C. I.; Istepanian, R. S. H.; Pantziaris, M.; and Nicolaides, A. (2005): Comparative evaluation of despeckle filtering in ultrasound imaging of the carotid artery. IEEE Trans. Ultrason. Ferroelectr. Freq. Control. 52(10), pp. 1653-1669.

[18] Gungor, A.; and Karagoz, I. (2016): Comparing Non-homomorphic and Homomorphic Wavelet Filtering Techniques for Speckled Images. International Journal of Computer Theory and Engineering, 8 (5), pp. 393-397.

[19] Zhou, Z.; Guo, Z.; Dong, G.; Sun, J.; Zhang, D.; and Wu, B. (2015): A Doubly Degenerate Diffusion Model Based on the Gray Level Indicator for Multiplicative Noise Removal. IEEE Transactions on Image Processing, 24(1), pp. $249-260$.

[20] Choi, H.; and Jeong, J. (2018). Speckle noise reduction in ultrasound images using SRAD and guided filter. 2018 International Workshop on Advanced Image Technology (IWAIT).

[21] Anthony Bua. (2021): Edge-aware nonlinear diffusion-driven regularization model, MATLAB Central File Exchange. https://www.mathworks.com/matlabcentral/fileexchange/74536-edge-aware-nonlinear-diffusion-driven-regularization-model (Retrieved April 1, 2021).

[22] Yu, Y.; and Acton, S.T. (2002): Speckle reducing anisotropic diffusion. IEEE Trans. Image Process. 11(11), pp. 1260-1270. 